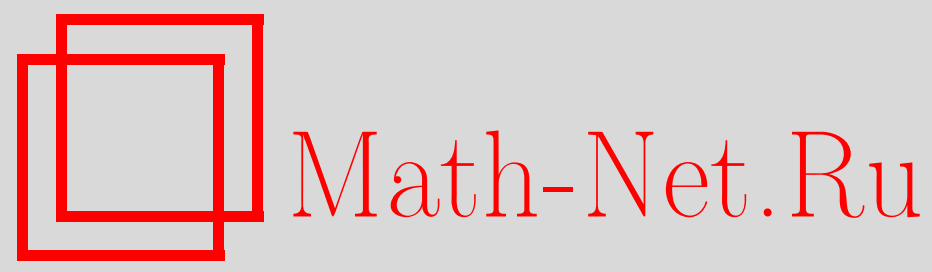

А. Г. Кузнецов, Производные категории трехмерных многообразий Фано $V_{12}$, Матем. заметки, 2005, том 78, выпуск 4, 579-594

DOI: https://doi.org/10.4213/mzm2615

Использование Общероссийского математического портала Math-Net.Ru подразумевает, что вы прочитали и согласны с пользовательским соглашением http://www.mathnet.ru/rus/agreement

Параметры загрузки:

IP : 54.205 .225 .156

26 апреля 2023 г., $18: 32: 44$

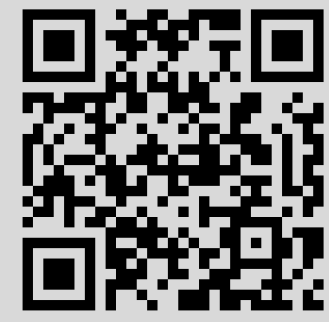


УДК 514.762

\title{
ПРОИЗВОДНЫЕ КАТЕГОРИИ ТРЕХМЕРНЫХ МНОГООБРАЗИЙ ФАНО $V_{12}$
}

\author{
А. Г. Кузнецов
}

В настоящей работе приводится описание производной категории когерентных пучков на трехмерном многообразии Фано индекса 1 и степени 12 (многообразии $V_{12}$ ). Легко показать, что если $X$ - многообразие $V_{12}$, то его производная категория содержит исключительную пару векторных расслоений $\left(\mathscr{U}, \mathscr{O}_{X}\right)$, где $\mathscr{O}_{X}-$ тривиальное расслоение, а $\mathscr{U}$ - мукаевское расслоение ранга 5 (индуцирующее вложение $X \rightarrow$ $\operatorname{Gr}(5,10))$. Ортогональную подкатегорию $\mathscr{A}_{X}={ }^{\perp}\langle\mathscr{U}, \mathscr{O}\rangle \subset \mathscr{D}^{b}(X)$ можно рассматривать как нетривиальную часть производной категории многообразия $X$. Основньмм результатом настоящей работы является конструкция эквивалентности категорий $\mathscr{A}_{X} \cong \mathscr{D}^{b}\left(C^{\vee}\right)$, где $C^{\vee}$ - кривая рода 7 , которая может быть канонически сопоставлена многообразию $X$ согласно результатам Илиева и Маркушевича. Для построения эквивалентности используются геометрические результаты Илиева и Маркушевича, а также результаты Бондала и Орлова о производных категориях. В качестве приложения доказывается, что поверхность Фано многообразия $X$ (поверхность, параметризующая коники на $X$ ) изоморфна $S^{2} C^{\vee}$ - симметрическому квадрату соответствующей кривой рода 7 .

Библиография: 10 названий.

Введение. Настоящая работа является продолжением серии работ [1], [2], посвященных описанию производных категорий когерентных пучков на трехмерных многообразиях $Ф$ ано. В данном случае объектом исследования являются многообразия типа $V_{12}$, которые согласно результатам Мукаи [3] можно представить в виде линейных сечений коразмерности 7 компоненты связности грассманиана $\operatorname{LGr}(5,10)$ пятимерных подпространств в $\mathbb{C}^{10}$, лагранжевых относительно фиксированной невырожденной квадратичной формы. Легко показать, что производная категория такого многообразия $X$ содержит исключительную пару векторных расслоений $\left(\mathscr{U}, \mathscr{O}_{X}\right)$, где $\mathscr{U}$ - ограничение тавтологического подрасслоения с $\operatorname{LGr}(5,10)$ на $X$. Вопрос, таким образом, сводится к описанию ортогонала

$$
\mathscr{A}_{X}:={ }^{\perp}\left\langle\mathscr{U}, \mathscr{O}_{X}\right\rangle \subset \mathscr{D}^{b}(X) .
$$

Работа выполнена при частичной поддержке Российского фонда фундаментальных исследований, гранты № 02-01-00468 и № 02-01-01041, гранта Президента РФ для поддержки молодых российских ученых, грант № MK-3926.2004.1, Американского фонда гражданских исследований CRDF, грант № RM1-2405-MO-02, а также Фонда содействия отечественной науке. 
Мы доказьваем, что на самом деле этот ортогонал эквивалентен производной категории кривой $C^{\vee}$ рода 7 , которая является ортогональным линейньм сечением (коразмерности 9) второй компоненты связности грассманиана $\operatorname{LGr}(5,10)$. Для построения эквивалентности используется результат Илиева и Маркушевича [4], утверждающий, что кривая $C^{\vee}$ является тонким многообразием модулей расслоений на $X$. Универсальное расслоение на $X \times C^{\vee}$ задает строго полньй функтор $\Phi$ урье-Мукаи $\Phi_{1}: \mathscr{D}^{b}\left(C^{\vee}\right) \rightarrow \mathscr{A}_{X}$.

Для доказательства того, что $\Phi_{1}$ - эквивалентность категорий, оказьвается удобно рассматривать пары, состоящие из многообразия $X$ и его гладкого гиперплоского сечения $S$. Легко видеть, что $S-K 3$-поверхность. Ортогональное линейное сечение второй компоненты связности грассманиана $\operatorname{LGr}(5,10)$ - еще одна $K 3$-поверхность $S^{\vee}$, содержащая кривую $C^{\vee}$ в качестве гиперплоского сечения. Согласно результатам Илиева и Маркушевича [4] поверхность $S^{\vee}$ также является тонким многообразием модулей расслоений на $S$. Универсальное расслоение на $S \times S^{\vee}$ задает строго полный функтор Фурье-Мукаи $\Phi_{2}: \mathscr{D}^{b}\left(S^{\vee}\right) \rightarrow \mathscr{D}^{b}(S)$, которьй оказывается эквивалентностью категорий вследствие замечания Бриджланда [5] о том, что всякий строго полный функтор между производньми категориями многообразий Калаби-Яу является эквивалентностью. Изучив связь функторов $\Phi_{1}$ и $\Phi_{2}$, удается вывести то, что функтор $\Phi_{1}$ - также эквивалентность.

В качестве применения полученного описания производной категории многообразия $X$ мы описьваем поверхность $\Phi$ ано, параметризующую коники на $X$. Оказывается, что эта поверхность изоморфна $S^{2} C^{\vee}$ - симметрическому квадрату соответствующей кривой рода 7. Для доказательства мы раскладываем структурный пучок произвольной коники по полученному полуортогональному разложению категории и замечаем, что его компонента в $\mathscr{D}^{b}\left(C^{\vee}\right)$ является структурньм пучком подсхемы длины два в $C^{\vee}$, что и дает искомый изоморфизм.

Приведем теперь краткое описание содержания данной работы. В п. 1 напоминаются основные результаты Мукаи и Илиева-Маркушевича о многообразиях $V_{12}$. В п. 2 исследуются универсальные расслоения на $X \times C^{\vee}$ и $S \times S^{\vee}$ и описывается их взаимосвязь. В п. 3 вычисляются когомологии некоторых расслоений. В п. 4 доказывается теорема о полуортогональном разложении категории $\mathscr{D}^{b}(X)$. В п. 5 приводится описание поверхности Фано многообразия $X$.

\section{1. Предварительные сведения.}

ОПРЕДЕЛЕНИЕ $1.1[6],[7]$. Многообразие $V_{12}$-этогладкое трехмерноемногообразие Фано $X$ индекса 1 с $\operatorname{Pic} X=\mathbb{Z}_{\text {и }}\left(-K_{X}\right)^{3}=12$.

Зафиксируем векторное пространство $V=\mathbb{C}^{10}$ и невырожденную квадратичную форму $Q$ на $V$. Обозначим через $S^{+} V$ и $S^{-} V$ спинорные (16-мерные) представления спинорной групшы $\operatorname{Spin}(Q)$. Напомним, что пространства $S^{ \pm} V$ совпадают с (двойственными) пространствами глобальных сечений обильных образующих групп Пикара связных компонент $\operatorname{LGr}_{ \pm}(V)$ лагранжева грассманиана пространства $V$ относительно $Q$. В частности, имеем канонические вложения $\operatorname{LGr}_{ \pm}(V) \rightarrow \mathbb{P}\left(S^{ \pm} V\right)$.

Выберем пару векторных подпространств $A_{8} \subset A_{9} \subset S^{+} V$ таких, что $\operatorname{dim} A_{i}=i$, и рассмотрим пересечения

$$
\begin{aligned}
& S=\mathrm{LGr}_{+}(V) \cap \mathbb{P}\left(A_{8}\right) \subset \mathbb{P}\left(S^{+} V\right), \\
& X=\mathrm{LGr}_{+}(V) \cap \mathbb{P}\left(A_{9}\right) \subset \mathbb{P}\left(S^{+} V\right) .
\end{aligned}
$$


Легко показать, что если $X$ гладко, то $X$ - многообразие $V_{12}$, а если $S$ гладко, то $S-$ поляризованная $K 3$-поверхность степени 12 .

Теорема 1.2 [3]. Если $X$-многообразие $V_{12}$, а $S \subset X-$ гладкая K3-поверхность, то существует такая пара векторных подпространств $A_{8} \subset A_{9} \subset S^{+} V$, что $S$ и $X$ получаются как в (1).

Напомним, что спинорные представления $S^{-} V$ и $S^{+} V$ канонически двойственны друг другу; обозначим через $B_{7} \subset B_{8} \subset S^{-} V$ ортогональные подпространства;

$$
B_{i}=A_{16-i}^{\perp} \subset S^{+} V^{*} \cong S^{-} V
$$

и рассмотрим двойственную пару

$$
\begin{aligned}
C^{\vee} & =\mathrm{LGr}_{-}(V) \cap \mathbb{P}\left(B_{7}\right) \subset \mathbb{P}\left(S^{-} V\right), \\
S^{\vee} & =\mathrm{LGr}_{-}(V) \cap \mathbb{P}\left(B_{8}\right) \subset \mathbb{P}\left(S^{-} V\right) .
\end{aligned}
$$

Как и выше, легко показать, что если $S^{\vee}$ гладко, то $S^{\vee}$ - поляризованная $K 3$-поверхность степени 12 , а если $C^{\vee}$ гладко, то $C^{\vee}$ - канонически вложенная кривая рода 7.

Обозначим через $H_{X}, L_{X}$ и $P_{X}$ классы гиперплоского сечения, прямой и точки в $H^{\bullet}(X, \mathbb{Z})$ соответственно. Аналогичные обозначения используются также для многообразий $S, S^{\vee}$ и $C^{\vee}$. Например, $P_{S} \vee \in H^{4}\left(S^{\vee}, \mathbb{Z}\right)$ обозначает класс точки на $S^{\vee}$.

Обозначим через $\mathscr{U}_{+}$и $\mathscr{U}_{-}$тавтологические подрасслоения на $\mathrm{LGr}_{+}(V) \subset \mathrm{Gr}(5, V)$ и $\mathrm{LGr}_{-}(V) \subset \mathrm{Gr}(5, V)$ соответственно, а через $\mathscr{U}_{+}$и $\mathscr{U}_{-}{ }_{y}-$ их слои в точках $x \in \mathrm{LGr}_{+}(V)$ и $y \in \mathrm{LGr}_{-}(V)$.

Напомним, как связана каноническая двойственность многообразий $\mathrm{LGr}_{ \pm}(V)$ с пересечением подпространств.

Лемма 1.3. Пусть $x \in \mathrm{LGr}_{+}(V) \subset \mathbb{P}\left(S^{-} V\right), y \in \operatorname{LGr}_{-}(V) \subset \mathbb{P}\left(S^{+} V\right)$, u обозначим через $\langle\cdot, \cdot\rangle-$ спаривание на $S^{+} V \times S^{-} V$, индуцирующее двойственность. Tогда

$$
\begin{aligned}
&\langle x, y\rangle \neq 0 \Longleftrightarrow \mathscr{U}_{+} \cap \mathscr{U}_{-}=0, \\
&\langle x, y\rangle=0 \Longleftrightarrow \operatorname{dim}\left(\mathscr{U}_{+} \cap \mathscr{U}_{-}\right) \geqslant 2 .
\end{aligned}
$$

Следовательно, для всех $(x, y) \in X \times C^{\vee}$ или $(x, y) \in S \times S^{\vee}$ имеем

$$
\operatorname{dim}\left(\mathscr{U}_{+x} \cap \mathscr{U}_{-y}\right) \geqslant 2
$$

ЛЕмма 1.4 [4]. Выполняются следующие әквивалентности:

(i) $C^{\vee}$ гладкая $\Longleftrightarrow X$ гладкое $\Longleftrightarrow$ для всех $x \in X, y \in C^{\vee}$ имеем $\operatorname{dim}\left(\mathscr{U}_{+_{x}} \cap \mathscr{U}_{-y}\right)=2$

(ii) $S^{\vee}$ гладкая $\Longleftrightarrow S$ гладкая $\Longleftrightarrow$ для всех $x \in S, y \in S^{\vee}$ имеем $\operatorname{dim}\left(\mathscr{U}_{+_{x}} \cap\right.$ $\left.\mathcal{U}_{-y}\right)=2$.

Следующая теорема объясняет внутренний смысл кривой $C^{\vee}$ и поверхности $S^{\vee}$ с точки зрения $X$ и $S$ соответственно. 
ТЕОремА 1.5 [4]. і) Кривая $C^{\vee}$ является тонким многообразием модулей стабильных расслоений $E$ ранга 2 на $X$ с классами Черна $c_{1}(E)=H_{X}, c_{2}(E)=5 L_{X}$. Если $E_{y}, E_{y^{\prime}}$ - расслоения на $X$, соответствующие точкам $y, y^{\prime} \in C^{\vee}$, то

$$
\operatorname{Ext}^{p}\left(E_{y}, E_{y^{\prime}}\right)= \begin{cases}\mathbb{C}, & \text { если } p=0,1 \text { и y= } y^{\prime} \\ 0, & \text { иначе. }\end{cases}
$$

ii) Поверхность $S^{\vee}$ является тонким многообразием модулей стабильных расслоений $E$ ранга 2 на $S$ с классами Черна $c_{1}(E)=H_{S}, c_{2}(E)=5 P_{S}$. Eсли $E_{y}$, $E_{y^{\prime}}-$ расслоения на $S$, соответствуюшие точкам $y, y^{\prime} \in S^{\vee}$, то

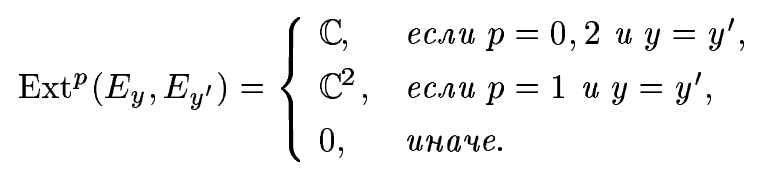

2. Универсальные расслоения. Рассмотрим одно из следующих произведений:

$$
W_{1}=X \times C^{\vee} \quad \text { или } \quad W_{2}=S \times S^{\vee} .
$$

Обозначим через $\mathscr{U}_{+}$и $\mathscr{U}_{-}$обратные образы тавтологических подрасслоений с $\mathrm{LGr}_{+}(V)$ и $\mathrm{LGr}_{-}(V)$ на $W_{i} \subset \mathrm{LGr}_{+}(V) \times \mathrm{LGr}_{-}(V)$ и рассмотрим следующие композиции естественных морфизмов векторных расслоений на $W_{i}$ :

$$
\xi_{i}: \mathscr{U}_{-} \rightarrow V \otimes \mathscr{O}_{W_{i}} \stackrel{Q}{=} V^{*} \otimes \mathscr{O}_{W_{i}} \rightarrow \mathscr{U}_{+}^{*} .
$$

Лемма 2.1. Если $X$ (соответственно $S$ ) гладко, то ранг $\xi_{1}$ (соответственно $\xi_{2}$ ) равен 3 во всякой точке $W_{1}$ (соответственно $W_{2}$ ).

ДокАЗАтЕльство. Ядро естественной сюръекции $V^{*} \otimes \mathscr{O}_{\mathrm{LGr}_{+}(V)} \rightarrow \mathscr{U}_{+}^{*}$ равно $\mathscr{U}_{+}$, поэтому достаточно показать, что для всех точек $(x, y) \in W_{i} \subset \mathrm{LGr}_{+}(V) \times \mathrm{LGr}_{-}(V)$ имеем $\operatorname{dim}\left(\mathscr{U}_{+} \cap \mathscr{U}_{-y}\right)=2$, что немедленно вытекает из леммы 1.3 .

Лемма 2.2. Имеем $\operatorname{Ker} \xi_{i} \cong\left(\operatorname{Coker} \xi_{i}\right)^{*}$.

ДокАЗАтЕльСтво. Рассмотрим следующую коммутативную диаграмму с точными строками на $W_{i}$ :

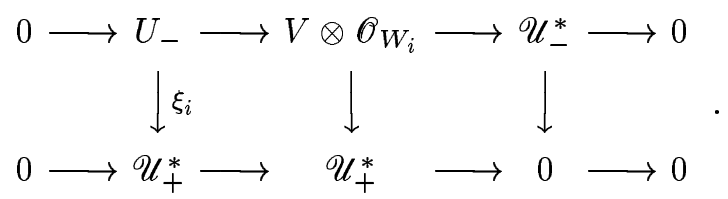

Заметим, что средняя вертикальная стрелка сюръективна, а ее ядро равно $\mathscr{U}_{+}$. Следовательно, длинная последовательность ядер и коядер имеет вид

$$
0 \rightarrow \operatorname{Ker} \xi_{i} \rightarrow \mathscr{U}_{+} \rightarrow \mathscr{U}_{-}^{*} \rightarrow \text { Coker } \xi_{i} \rightarrow 0 .
$$

Далее, легко видеть, что отображение $\mathscr{U}_{+} \rightarrow \mathscr{U}_{-}^{*}$ в этой последовательности двойственно к отображению $\xi_{i}^{*}$. Отсюда немедленно вытекает, что $\operatorname{Ker} \xi_{i} \cong \operatorname{Ker}\left(\xi_{i}^{*}\right) \cong\left(\operatorname{Coker} \xi_{i}\right)^{*}$.

Обозначим через $\mathscr{E}_{i}$ коядро морфизма $\xi_{i}$ на $W_{i}$. Из леммы 2.1 следует, что $\mathscr{E}_{i}$ является расслоением ранга 2 на $W_{i}$, и имеет место следующая точная последовательность:

$$
0 \rightarrow \mathscr{E}_{i}^{*} \rightarrow \mathscr{U}_{-} \stackrel{\xi_{i}}{\rightarrow} \mathscr{U}_{+}^{*} \rightarrow \mathscr{E}_{i} \rightarrow 0 .
$$

Дуализируя, получаем еще одну последовательность:

$$
0 \rightarrow \mathscr{E}_{i}^{*} \rightarrow \mathscr{U}_{+} \stackrel{\xi_{\dot{\rightarrow}}^{*}}{\rightarrow} \mathscr{U}_{-}^{*} \rightarrow \mathscr{E}_{i} \rightarrow 0 .
$$


Лемма 2.3. Классы Черна расслоений $\mathscr{E}_{i}$ имеют вид

$$
\begin{aligned}
\mathrm{c}_{1}\left(\mathscr{E}_{1}\right) & =H_{X}+H_{C \vee}, & \mathrm{c}_{2}\left(\mathscr{E}_{1}\right) & =\frac{7}{12} H_{X} H_{C}+5 L_{X}+\eta, \\
\mathrm{c}_{1}\left(\mathscr{E}_{2}\right) & =H_{S}+H_{S \vee}, & \mathrm{c}_{2}\left(\mathscr{E}_{2}\right) & =\frac{7}{12} H_{S} H_{S} \vee 5 P_{S}+5 P_{S} \vee
\end{aligned}
$$

əде

$$
\eta \in\left(H^{3}(X, \mathbb{C}) \otimes H^{1}\left(C^{\vee}, \mathbb{C}\right)\right) \cap H^{4}\left(X \times C^{\vee}, \mathbb{Z}\right)
$$

ДокАЗАТЕЛЬСТво. Из (3) следует, что

$$
\operatorname{ch}\left(\mathscr{U}_{+}^{*}\right)-\operatorname{ch}\left(\mathscr{U}_{-}\right)=\operatorname{ch}\left(\mathscr{E}_{i}\right)-\operatorname{ch}\left(\mathscr{E}_{i}^{*}\right)=2 \operatorname{ch}_{1}\left(\mathscr{E}_{i}\right)+2 \operatorname{ch}_{3}\left(\mathscr{E}_{i}\right) .
$$

Отсюда получаем

$$
\begin{aligned}
\operatorname{ch}_{1}\left(\mathscr{E}_{1}\right) & =H_{X}+H_{C \vee}, \quad \operatorname{ch}_{3}\left(\mathscr{E}_{1}\right)=-\frac{1}{2} P_{X}, \\
\operatorname{ch}_{1}\left(\mathscr{E}_{2}\right) & =H_{S}+H_{S \vee}, \quad \operatorname{ch}_{3}\left(\mathscr{E}_{2}\right)=-\frac{1}{2} P_{S}-\frac{1}{2} P_{S} \vee
\end{aligned}
$$

Ясно, что $c_{1}\left(\mathscr{E}_{i}\right)=\operatorname{ch}_{1}\left(\mathscr{E}_{i}\right)$. Кроме того, из формулы Кюннета следует, что

$$
\mathrm{c}_{2}\left(\mathscr{E}_{1}\right)=a_{1} H_{X} H_{C} \vee+b_{1} L_{X}+\eta, \quad c_{2}\left(\mathscr{E}_{2}\right)=a_{2} H_{S} H_{S \vee}+b_{2} P_{S}+c_{2} P_{S} \vee
$$

где $a_{1}, b_{1}, a_{2}, b_{2}, c_{2} \in \mathbb{Q}, \eta \in\left(H^{3}(X, \mathbb{C}) \otimes H^{1}\left(C^{\vee}, \mathbb{C}\right)\right) \cap H^{4}\left(X \times C^{\vee}, \mathbb{Z}\right)$. Поскольку соответствие $S \leftrightarrow S^{\vee}$ симметрично, заключаем, что $c_{2}=b_{2}$. Наконец, числа $a_{i}$ и $b_{i}$ можно вычислить из равенства

$$
3 c_{1}\left(\mathscr{E}_{i}\right) \mathrm{c}_{2}\left(\mathscr{E}_{i}\right)=\operatorname{ch}_{1}\left(\mathscr{E}_{i}\right)^{3}-6 \operatorname{ch}_{3}\left(\mathscr{E}_{i}\right)
$$

ЗАмечание 2.4. Пользуясь формулой Римана-Роха на $X \times C^{\vee}$, можно вычислить, что $\eta^{2}=14$.

СлЕДСТВИЕ 2.5. Расслоение $\mathscr{E}_{1}$ (соответственно $\left.\mathscr{E}_{2}\right)$ является универсальным расслоением ранга 2 с $\mathrm{c}_{1}=\left[H_{X}\right], \mathrm{c}_{2}=5\left[L_{X}\right]$ на $X$ (соответственно с $\mathrm{c}_{1}=\left[H_{S}\right]$, $\mathrm{c}_{2}=5\left[P_{S}\right]$ на $\left.S\right)$.

ДокАЗАТЕЛЬСтво. Для всякой точки $y \in C^{\vee}$ (соответственно $y \in S^{\vee}$ ) обозначим через $\mathscr{E}_{1 y}$ ограничение $\mathscr{E}_{1}$ на $X \times y$ и через $\mathscr{E}_{2 y}$ - ограничение $\mathscr{E}_{2}$ на $S \times y$. В леммах 3.3 и 3.5 будет показано, что расслоения $\mathscr{E}_{1 y}$ на $X$ при всех $y \in C^{\vee}$, а также расслоения $\mathscr{E}_{2 y}$ на $S$ при всех $y \in S^{\vee}$ стабильны; следовательно, существуют морфизмы

$$
f_{1}: C^{\vee} \rightarrow \mathscr{M}_{X}\left(2,\left[H_{X}\right], 5\left[L_{X}\right]\right), \quad f_{2}: S^{\vee} \rightarrow \mathscr{M}_{S}\left(2,\left[H_{S}\right], 5\left[P_{S}\right]\right),
$$

в многообразия модулей расслоений ранга 2 на $X$ и $S$ соответственно с указанными классами Черна такие, что

$$
\mathscr{E}_{1}=\left(\mathrm{id}_{X} \times f_{1}\right)^{*} \mathscr{E}_{1}^{\prime} \otimes q_{1}^{*} \mathscr{L}_{1}, \quad \mathscr{E}_{2}=\left(\operatorname{id}_{S} \times f_{2}\right)^{*} \mathscr{E}_{2}^{\prime} \otimes q_{2}^{*} \mathscr{L}_{2},
$$


где $\mathscr{E}_{1}^{\prime}$ и $\mathscr{E}_{2}^{\prime}$ - универсальные расслоения на $X \times \mathscr{M}_{X}\left(2,\left[H_{X}\right], 5\left[L_{X}\right]\right)$ и $S \times \mathscr{M}_{S}\left(2,\left[H_{S}\right]\right.$, $\left.5\left[P_{S}\right]\right)$ соответственно, $q_{1}: X \times C^{\vee} \rightarrow C^{\vee}$ и $q_{2}: S \times S^{\vee} \rightarrow S^{\vee}$ - проекции, а $\mathscr{L}_{1}$ и $\mathscr{L}_{2}-$ линейные расслоения на $C^{\vee}$ и $S^{\vee}$.

Легко видеть, что морфизмы $f_{1}$ и $f_{2}$ совпадают с отображениями $\rho$, построенными в $[4$, раздел 4$]$. Следовательно, они являются изоморфизмами, а расслоения $\mathscr{E}_{1}$ и $\mathscr{E}_{2}$ являются универсальными расслоениями.

Обозначим через

$$
\alpha: S \rightarrow X \quad \text { и } \quad \beta: C^{\vee} \rightarrow S^{\vee}
$$

вложения, и пусть

$$
\lambda_{1}=\alpha \times \operatorname{id}_{C^{\vee}}, \quad \lambda_{2}=\operatorname{id}_{S} \times \beta, \quad \mu_{1}=\operatorname{id}_{X} \times \beta, \quad \mu_{2}=\alpha \times \operatorname{id}_{S^{\vee}}, \quad \nu=\alpha \times \beta .
$$

Получаем коммутативную диаграмму

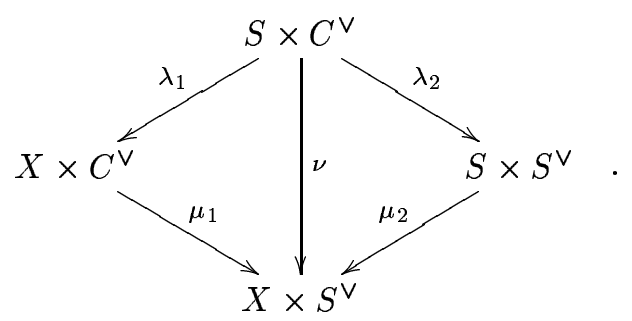

ЛЕмма 2.6. Имеем канонические изоморфизмы $\lambda_{1}^{*} \mathscr{E}_{1}=\lambda_{2}^{*} \mathscr{E}_{2}$.

ДокАЗАТЕЛЬСтво. Утверждение очевидно, поскольку $\lambda_{i}^{*} \mathscr{E}_{i}$ является коядром морфизма $\lambda_{i}^{*} \xi_{i}$, а $\lambda_{1}^{*} \xi_{1}=\lambda_{2}^{*} \xi_{2}$ по определению морфизмов $\xi_{i}$.

Обозначим расслоение $\lambda_{1}^{*} \mathscr{E}_{1}=\lambda_{2}^{*} \mathscr{E}_{2}$ на $S \times C^{\vee}$ через $\mathscr{E}$.

Рассмотрим произведение $\widetilde{W}=X \times S^{\vee}$ и композицию гомоморфизмов

$$
\tilde{\xi}: \mathscr{U}_{i} \rightarrow V \otimes \mathscr{O}_{\widetilde{W}} \stackrel{Q}{\cong} V^{*} \otimes \mathscr{O}_{\widetilde{W}} \rightarrow \mathscr{U}_{+}^{*}
$$

Ясно, что

$$
\mu_{i}^{*} \tilde{\xi}=\xi_{i}
$$

Лемма 2.7. Ранг морфизма $\tilde{\xi}$ на $X \times S^{\vee} \backslash\left(\mu_{1}\left(X \times C^{\vee}\right) \cup \mu_{2}\left(S \times S^{\vee}\right)\right)$ равен 5 .

ДоКАЗАТЕЛЬСТВо следует из леммы 1.3.

Обозначим через $\widetilde{\mathscr{E}}$ коядро морфизма $\tilde{\xi}$.

ЛЕмма 2.8. Имеем точные последовательности на $X \times S^{\vee}$

$$
0 \rightarrow \mathscr{U}_{-} \stackrel{\tilde{\xi}}{\rightarrow} \mathscr{U}_{+}^{*} \rightarrow \widetilde{\mathscr{E}} \rightarrow 0, \quad 0 \rightarrow \widetilde{\mathscr{E}} \rightarrow \mu_{1_{*}} \mathscr{E} 1 \oplus \mu_{2 *} \mathscr{E}_{2} \rightarrow \nu_{*} \mathscr{E} \rightarrow 0
$$


ДокАЗАТЕльСтво. Первая последовательность точна по лемме 2.7 и определению $\widetilde{\mathscr{E}}$. Чтобы проверить точность второй последовательности, заметим, что $\mu_{i}^{*} \widetilde{\mathscr{E}}=\mathscr{E}_{i}$ по (6) и $(3)$ и что канонические сюръекции $\widetilde{\mathscr{E}} \rightarrow \mu_{i_{*}} \mu_{i}^{*} \widetilde{\mathscr{E}}=\mu_{i_{*}} \mathscr{E}_{i}$ склеиваются в сюръекцию

$$
\widetilde{\mathscr{E}} \rightarrow \operatorname{Ker}\left(\mu_{1 *} \mathscr{E}_{1} \oplus \mu_{2 *} \mathscr{E}_{2} \rightarrow \nu_{*} \mathscr{E}\right)
$$

С другой стороны, легко проверить, что характеры Черна пучков $\widetilde{\mathscr{E}}$ и $\operatorname{Ker}\left(\mu_{1 *} \mathscr{E}_{1} \oplus \mu_{2} \mathscr{E}_{2}\right.$ $\left.\rightarrow \nu_{*} \mathscr{E}\right)$ совпадают. Следовательно,

$$
\widetilde{\mathscr{E}} \cong \operatorname{Ker}\left(\mu_{1_{*}} \mathscr{E} 1 \oplus \mu_{2} \mathscr{E}_{2} \rightarrow \nu_{*} \mathscr{E}\right)
$$

и доказательство закончено.

СлЕДСТВИЕ 2.9. Имеем следующую точную последовательность на $X \times S^{\vee}$ :

$$
0 \rightarrow \mu_{1_{*}} \mathscr{E}_{1} \otimes \mathscr{O}\left(-H_{X}\right) \rightarrow \widetilde{\mathscr{E}} \rightarrow \mu_{2 *} \mathscr{E}_{2} \rightarrow 0
$$

\section{3. Вычисление когомологий.}

ЛЕмма 3.1. Выполнены равенства

$$
\begin{aligned}
\operatorname{Ext}^{k}\left(\mathscr{U}_{+}, \mathscr{U}_{+}\right) & =H^{k}\left(X, \mathscr{U}_{+}^{*} \otimes \mathscr{U}_{+}\right)=\operatorname{Ext}^{k}\left(\mathscr{O}_{X}, \mathscr{O}_{X}\right) \\
& =H^{k}\left(X, \mathscr{O}_{X}\right)= \begin{cases}\mathbb{C} & n p u k=0, \\
0 & n p u k \neq 0,\end{cases} \\
\operatorname{Ext}^{\bullet}\left(\mathscr{O}_{X}, \mathscr{U}_{+}\right) & =H^{\bullet}\left(X, \mathscr{U}_{+}\right)=0 .
\end{aligned}
$$

ДокАЗАТЕЛЬСтво. Напомним, что $X$ является полным пересечением $\mathbb{P}\left(A_{9}\right)$ $\cap \mathrm{LGr}_{+}(V) \subset \mathbb{P}(S P V)$ и $S^{+} V / A_{9}=B_{7}^{*}$. Поэтому $X \subset \mathrm{LGr}_{+}(V)$ является схемой нулей сечения расслоения $B_{7}^{*} \otimes \mathscr{O}_{\mathrm{LGr}_{+}(V)}\left(H_{\mathrm{LGr}_{+}(V)}\right)$. Значит, комплекс Кошуля $\Lambda^{\bullet}\left(B_{7}^{*} \otimes\right.$ $\left.\mathscr{O}_{\mathrm{LGr}_{+}(V)}\left(H_{\mathrm{LGr}_{+}(V)}\right)\right)$ является резольвентой структурного пучка $\mathscr{O}_{X}$ на $\mathrm{LGr}_{+}(V)$. Иначе говоря, имеем точную последовательность

$$
\begin{aligned}
0 & \rightarrow \Lambda^{7}\left(B_{7} \otimes \mathscr{O}_{\mathrm{LGr}_{+}(V)}\left(-H_{\mathrm{LGr}_{+}(V)}\right)\right) \rightarrow \cdots \rightarrow B_{7} \otimes \mathscr{O}_{\mathrm{LGr}_{+}(V)}\left(-H_{\mathrm{LGr}_{+}(V)}\right) \\
& \rightarrow \mathscr{O}_{\mathrm{LGr}_{+}(V) \rightarrow \mathscr{O}_{X} \rightarrow 0 .}
\end{aligned}
$$

Домножая ее на $\mathscr{U}_{+}$или $\mathscr{U}_{+}^{*} \otimes \mathscr{U}_{+}$и вычисляя когомологии возникающих эквивариантных расслоений на $\mathrm{LGr}_{+}(V)$ по теореме Бореля-Ботта-Вейля [8], получаем искомые равенства.

Поскольку канонический класс $X$ равен $-H_{X}$, по двойственности Серра получаем

СЛЕДСТВИЕ 3.2. Имеем

$$
H^{\bullet}\left(X, \mathscr{U}_{+}^{*}\left(-H_{X}\right)\right)=0, \quad H^{k}\left(X, \mathscr{U}_{+} \otimes \mathscr{U}_{+}^{*}\left(-H_{X}\right)\right)= \begin{cases}\mathbb{C} & n p u k=3, \\ 0 & n p u k \neq 3\end{cases}
$$


ЛЕмма 3.3. При всех $y \in C^{\vee}$ имеем

$$
H^{p}\left(X, \mathscr{E}_{1 y}\left(-H_{X}\right)\right)=H^{p}\left(X, \mathscr{E}_{1 y} \otimes \mathscr{U}_{+}^{*}\left(-H_{X}\right)\right)=0
$$

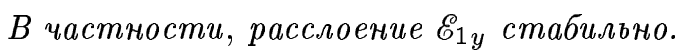

ДокАЗАТЕЛЬСтво. Напомним, что по определению $C^{\vee}=\mathrm{LGr}_{-}(V) \cap \mathbb{P}\left(B_{7}\right)$, a $X=$ $\mathrm{LGr}_{+}(V) \cap \mathbb{P}\left(A_{9}\right)$, где $A_{9}=B_{7}^{\perp}$. Выберем 6-мерное подпространство $B_{6} \subset B_{7}$ такое, что $\mathbb{P}\left(B_{6}\right)$ пересекает $C^{\vee}$ трансверсально и не содержит $y$. Положим $A_{10}=B_{6}^{\perp}$ и рассмотрим $\widehat{X}=\mathrm{LGr}_{+}(V) \cap \mathbb{P}\left(A_{10}\right)$. Рассуждения леммы 1.4 показьвают, что $\widehat{X}$ - гладкое четырехмерное многообразие $\Phi$ ано индекса 2 , содержащее $X$ как гиперплоское сечение. Более того, рассуждения, аналогичные использованньп в лемме 2.8, показьвают, что композиция

$$
\hat{\xi}: \mathscr{U}_{-y} \otimes \mathscr{O}_{\widehat{X}} \rightarrow V \otimes \mathscr{O}_{\widehat{X}} \rightarrow \mathscr{U}_{+}^{*}
$$

инъективна, а ее коядро изоморфно прямому образу расслоения $\mathscr{E}_{1 y}$ при вложении $i$ : $X \rightarrow \widehat{X}$. Иначе говоря, мы имеем следующую точную последовательность на $\widehat{X}$ :

$$
0 \rightarrow \mathscr{U}_{-y} \otimes \mathscr{O}_{\widehat{X}} \rightarrow \mathscr{U}_{+}^{*} \rightarrow i_{*} \mathscr{E}_{1 y} \rightarrow 0
$$

С другой стороны, пользуясь теоремой Бореля-Ботта-Вейля и резольвентой Кошуля многообразия $\widehat{X} \subset \mathrm{LGr}_{+}(V)$, можно вьчислить

$$
H^{\bullet}\left(\widehat{X}, \mathscr{U}_{+}^{*} \otimes \mathscr{U}_{+}^{*}\left(-H_{\widehat{X}}\right)\right)=H^{\bullet}\left(\widehat{X}, \mathscr{U}_{+}^{*}\left(-H_{\widehat{X}}\right)\right)=H^{\bullet}\left(\widehat{X}, \mathscr{O}_{\widehat{X}}\left(-H_{\widehat{X}}\right)\right)=0
$$

и лемма следует из последовательностей когомологий, возникающих из точной последовательности $(7)$, подкрученной на $\mathscr{O}_{\widehat{X}}\left(-H_{\widehat{X}}\right)$ и $\mathscr{U}_{+}^{*}\left(-H_{\widehat{X}}\right)$ соответственно, поскольку

$$
\begin{aligned}
H^{\bullet}\left(X, \mathscr{E}_{1 y}\left(-H_{X}\right)\right) & =H^{\bullet}\left(\widehat{X}, i_{*} \mathscr{E}_{1 y}\left(-H_{\widehat{X}}\right)\right), \\
H^{\bullet}\left(X, \mathscr{E}_{1 y} \otimes \mathscr{U}_{+}^{*}\left(-H_{X}\right)\right) & =H^{\bullet}\left(\widehat{X}, i_{*} \mathscr{E}_{1 y} \otimes \mathscr{U}_{+}^{*}\left(-H_{\widehat{X}}\right)\right) .
\end{aligned}
$$

ЛЕмма 3.4. При всех $y \in C^{\vee}$ имеем

$$
H^{1}\left(X, \mathscr{E}_{1 y}\left(-2 H_{X}\right)\right)=0
$$

ДоКАЗАТЕЛЬСТво. Ограничивая точную последовательность (3) на $X=X \times\{y\} \subset$ $X \times C^{\vee}$, подкручивая ее на $\mathscr{O}_{X}\left(-H_{X}\right)$ и учитьвая лемму 2.3 , получаем точную последовательность

$$
0 \rightarrow \mathscr{E}_{1 y}\left(-2 H_{X}\right) \rightarrow \mathscr{U}_{-y} \otimes \mathscr{O}_{X}\left(-H_{X}\right) \rightarrow \mathscr{U}_{+y}^{*}\left(-H_{X}\right) \rightarrow \mathscr{E}_{1 y}\left(-H_{X}\right) \rightarrow 0
$$

Из следствия 3.2 и леммы 3.3 вытекает, что

$$
H^{1}\left(X, \mathscr{E}_{1 y}\left(-2 H_{X}\right)\right) \cong \mathscr{U}_{-y} \otimes H^{1}\left(X, \mathscr{O}_{X}\left(-H_{X}\right)\right)
$$

Далее по двойственности Серра получаем

$$
H^{1}\left(X, \mathscr{O}_{X}\left(-H_{X}\right)\right)=H^{2}\left(X, \mathscr{O}_{X}\right)^{*},
$$

что равно нулю по лемме 3.1 . 
ЛЕмма 3.5. При всех $y \in S^{\vee}$ имеем

$$
H^{0}\left(S, \mathscr{E}_{2 y}\left(-H_{S}\right)\right)=0
$$

В частности, расслоение $\mathscr{E}_{2 \text { y }}$ стабильно.

ДокАЗАТЕльСТво. Если $y \in C^{\vee}$, то $\mathscr{E}_{2 y}=\mathscr{E}_{1 y}, S$, и лемма вытекает из точной последовательности

$$
H^{0}\left(X, \mathscr{E}_{1 y}\left(-H_{X}\right)\right) \rightarrow H^{0}\left(S, \mathscr{E}_{2 y}\left(-H_{S}\right)\right) \rightarrow H^{1}\left(X, \mathscr{E}_{1 y}\left(-2 H_{X}\right)\right),
$$

поскольку первьй член равен 0 по лемме 3.3 , а третий - по лемме 3.4 .

Теперь заметим, что, зафиксировав $S$ (а следовательно, и $S^{\vee}$ ) и варьируя $B_{7}$, мы можем получить в качестве $C^{\vee}$ любое гладкое гиперплоское сечение поверхности $S^{\vee}$ и рассмотреть соответствующее ему гладкое многообразие $X \supset S$. Поскольку всякая точка $y \in S^{\vee}$ лежит на гладком гиперплоском сечении, приведенные вьше рассуждения в применении к соответствующему $X$ доказывают лемму.

СлЕДСТВИЕ 3.6. При всех $y \in C^{\vee}$ имеем

$$
H^{p}\left(X, \mathscr{E}_{1 y} \otimes \mathscr{U}_{+}\left(-H_{X}\right)\right)=0
$$

ДоКАЗАТЕЛЬСТВО. Домножим точную последовательность

$$
0 \rightarrow \mathscr{U}_{+} \rightarrow V \otimes \mathscr{O}_{X} \rightarrow \mathscr{U}_{+}^{*} \rightarrow 0
$$

на $\mathscr{E}_{1 y}\left(-H_{X}\right)$ и рассмотрим соответствующую последовательность когомологий.

4. Производные категории. Рассмотрим функторы Фурье-Мукаи с ядрами $\mathscr{E}_{1}$ и $\mathscr{E}_{2}$ :

$$
\begin{gathered}
\Phi_{1}: \mathscr{D}^{b}\left(C^{\vee}\right) \rightarrow \mathscr{D}^{b}(X), \quad \Phi_{2}: \mathscr{D}^{b}\left(S^{\vee}\right) \rightarrow \mathscr{D}^{b}(S), \\
\Phi_{i}(-)=R p_{i_{*}}\left(L q_{i}{ }^{*}(-) \otimes \mathscr{E}_{i}\right),
\end{gathered}
$$

где $p_{i}$ и $q_{i}-$ проекции на первьй и второй сомножители:
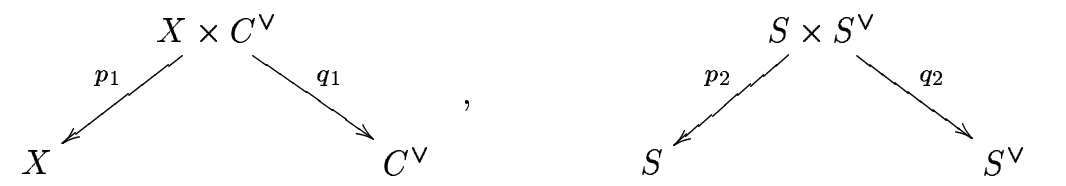

ТЕОРемА 4.1. Функторы $\Phi_{i}$ строго полны.

ДокАЗАТЕЛЬСтво. Согласно результатам Бондала и Орлова [9] достаточно проверить, что для структурных пучков любых двух точек $y_{1}, y_{2} \in C^{\vee}$ (соответственно $\left.y_{1}, y_{2} \in S^{\vee}\right)$ и всех $p \in \mathbb{Z}$ имеем

$$
\operatorname{Ext}^{p}\left(\Phi_{i}\left(\mathscr{O}_{y_{1}}\right), \Phi_{i}\left(\mathscr{O}_{y_{2}}\right)\right)=\operatorname{Ext}^{p}\left(\mathscr{O}_{y_{1}}, \mathscr{O}_{y_{2}}\right)
$$

С другой стороны, ясно, что $\Phi_{i}\left(\mathscr{O}_{y_{k}}\right)=\mathscr{E}_{i y_{k}}$, и остается использовать следствие 2.5 и теорему 1.5 . 
СлЕДСТВИЕ 4.2. Функтор $\Phi_{2}: \mathscr{D}^{b}\left(S^{\vee}\right) \rightarrow \mathscr{D}^{b}(S)$ - әквивалентность категорий.

ДокАЗАтЕльСтво. Согласно результатам Бриджланда любой строго полный функтор между производными категориями $K 3$-поверхностей является эквивалентностью [5].

Рассмотрим диаграмму

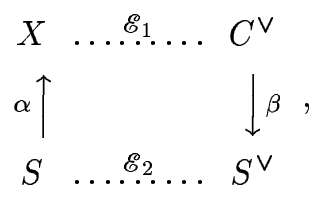

где пунктирная линия, соединяющая два многообразия, означает, что мы рассматриваем соответствующее ядро на их произведении. Из этой диаграммы возникает диаграмма функторов

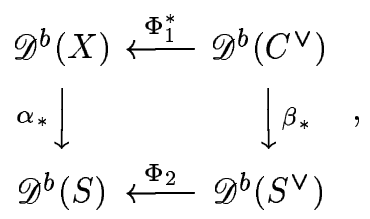

которая коммутативна по лемме 2.6 , поскольку функтор $\alpha^{*} \circ \Phi_{1}$ задается ядром $\lambda_{1}^{*} \mathscr{E} 1$, a функтор $\Phi_{2} \circ \beta_{*}-$ ядром $\lambda_{2}^{*} \mathscr{E}_{2}$.

Обозначим через

$$
\Phi_{1}^{*}: \mathscr{D}^{b}(X) \rightarrow \mathscr{D}^{b}\left(C^{\vee}\right) \quad \text { и } \quad \Phi_{2}^{*}: \mathscr{D}^{b}(S) \rightarrow \mathscr{D}^{b}\left(S^{\vee}\right)
$$

левые сопряженные функторы. Стандартные вычисления показьвают, что эти функторы задаются ядрами

$$
\begin{array}{ll}
\mathscr{E}_{1}^{*}\left(-H_{X}\right)[3]=\mathscr{E}_{1}\left(-2 H_{X}-H_{C^{\vee}}\right)[3] & \text { на } X \times C^{\vee}, \\
\mathscr{E}_{2}^{*}[2]=\mathscr{E}_{2}\left(-H_{S}-H_{S} \vee\right)[2] & \text { на } S \times S^{\vee}
\end{array}
$$

соответственно.

Рассмотрим теперь диаграмму

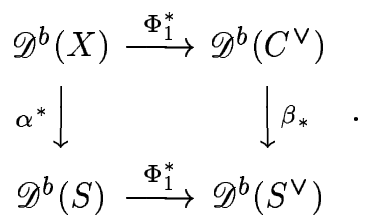

Она уже не является коммутативной. Однако следующее предложение показывает, что она становится коммутативной при замене категории $\mathscr{D}^{b}(X)$ подкатегорией ${ }^{\perp}\left\langle\mathscr{U}_{+}, \mathscr{O}_{X}\right\rangle$ $\subset \mathscr{D}^{b}(X)$.

ПРЕДЛОЖЕНИЕ 4.3. ФунКторы

$$
\beta_{*} \circ \Phi_{1}^{*} \quad u \quad \Phi_{2}^{*} \circ \alpha^{*}: \mathscr{D}^{b}(X) \rightarrow \mathscr{D}^{b}\left(S^{\vee}\right)
$$

изоморфны на подкатегории ${ }^{\perp}\left\langle\mathscr{U}_{+}, \mathscr{O}_{X}\right\rangle \subset \mathscr{D}^{b}(X)$. 
ДокАЗАТЕЛЬСтво. Легко видеть, что функторы $\beta_{*} \circ \Phi_{1}^{*}$ и $\Phi_{2}^{*} \circ \alpha^{*}$ задаются ядрами

$$
\mu_{1 *} \mathscr{E}_{1}\left(-2 H_{X}-H_{S}\right)[3] \quad \text { и } \quad \mu_{2 *} \mathscr{E}_{2}\left(-H_{X}-H_{S^{\vee}}\right)[2]
$$

соответственно на $X \times S^{\vee}$. Рассматривая спираль точной последовательности из следствия 2.9 , подкрученную на $\mathscr{O}\left(-H_{X}-H_{S} \vee\right)$, мы видим, что существует выделенный треугольник

$$
\mu_{2 *} \mathscr{E}_{2}\left(-H_{X}-H_{S \vee}\right)[2] \rightarrow \mu_{1_{*}} \mathscr{E}_{1}\left(-2 H_{X}-H_{S \vee}\right)[3] \rightarrow \widetilde{\mathscr{E}}\left(-H_{X}-H_{S \vee}\right)[3]
$$

Остается показать, что ядерный функтор $\mathscr{D}^{b}(X) \rightarrow \mathscr{D}^{b}\left(S^{\vee}\right)$, заданньй ядром $\widetilde{\mathscr{E}}\left(-H_{X}\right.$ $\left.-H_{S \vee}\right)$ зануляется на триангулированной подкатегории ${ }^{\perp}\left\langle\mathscr{U}_{+}, \mathscr{O}_{X}\right\rangle \subset \mathscr{D}^{b}(X)$.

Заметим, что из леммы 2.8 следует, что пучок $\widetilde{\mathscr{E}}\left(-H_{X}-H_{S}\right.$ ) изоморфен конусу морфизма

$$
\mathscr{U}_{-}\left(-H_{X}-H_{S} \vee\right) \rightarrow \mathscr{U}_{+}^{*}\left(-H_{X}-H_{S^{\vee}}\right)
$$

на $X \times S^{\vee}$, так что достаточно проверить, что ядерные функторы $\mathscr{D}^{b}(X) \rightarrow \mathscr{D}^{b}\left(S^{\vee}\right)$, заданные ядрами

$$
\mathscr{U}_{-}\left(-H_{X}-H_{S} \vee\right) \quad \text { и } \quad \mathscr{U}_{+}^{*}\left(-H_{X}-H_{S} \vee\right)
$$

на $X \times S^{\vee}$, зануляются на триангулированной подкатегории ${ }^{\perp}\left\langle\mathscr{U}_{+}, \mathscr{O}_{X}\right\rangle \subset \mathscr{D}^{b}(X)$. Обозначим через

$$
\tilde{p}: X \times S^{\vee} \rightarrow X \quad \text { и } \quad \tilde{q}: X \times S^{\vee} \rightarrow S^{\vee}
$$

проекции. Прямое вычисление с использованием формулы проекции и двойственности Серра на $X$ показывает, что для всякого объекта $F \in \mathscr{D}^{b}(X)$ имеем

$$
\begin{aligned}
\Phi_{\mathscr{U}_{-}\left(-H_{X}-H_{S} \vee\right.}(F) & =R \tilde{q}_{*}\left(L \tilde{p}^{*}(F) \otimes \mathscr{U}_{-}\left(-H_{X}-H_{S} \vee\right)\right) \\
& =\operatorname{R} \Gamma\left(X, F\left(-H_{X}\right)\right) \otimes \mathscr{U}_{-}\left(-H_{S} \vee\right. \\
& =\operatorname{RHom}\left(F, \mathscr{O}_{X}\right)^{*} \otimes \mathscr{U}_{-}\left(-H_{S} \vee\right), \\
\Phi_{\mathscr{U}_{+}^{*}\left(-H_{X}-H_{S} \vee\right.}(F) & =R \tilde{q}_{*}\left(L \tilde{p}^{*}(F) \otimes \mathscr{U}_{+}^{*}\left(-H_{X}-H_{S} \vee\right)\right) \\
& =\mathrm{R} \Gamma\left(X, F \otimes \mathscr{U}_{+}^{*}\left(-H_{X}\right)\right) \otimes \mathscr{O}_{S}\left(-H_{S} \vee\right) \\
& =\operatorname{RHom}\left(F, \mathscr{U}_{+}\right)^{*} \otimes \mathscr{O}_{S} \vee\left(-H_{S} \vee\right) .
\end{aligned}
$$

В частности, интересующие нас ядерные функторы равны нулю на всяком объекте $F \in$ ${ }^{\perp}\left\langle\mathscr{U}_{+}, \mathscr{O}_{X}\right\rangle \subset \mathscr{D}^{b}(X)$.

ТЕОРема 4.4. Имеем полуортогональное разложение

$$
\mathscr{D}^{b}(X)=\left\langle\mathscr{U}_{+}, \mathscr{O}_{X}, \Phi_{1}\left(\mathscr{D}^{b}\left(C^{\vee}\right)\right)\right\rangle
$$


ДокАЗАТЕЛЬСТвО. По лемме 3.1 пара расслоений $\left(\mathscr{U}_{+}, \mathscr{O}_{X}\right)$ исключительная. Более того, обозначая через $\Phi_{1}^{!}: \mathscr{D}^{b}(X) \rightarrow \mathscr{D}^{b}\left(C^{\vee}\right)$ правьй сопряженный функтор и подставляя $F=\mathscr{O}_{X}$ или $F=\mathscr{U}_{+}$, мы видим, что

$$
\begin{aligned}
\operatorname{Ext}^{\bullet}\left(\mathscr{O}_{y}, \Phi_{1}^{!}(F)\right) & =\operatorname{Ext}^{\bullet}\left(\Phi_{1}\left(\mathscr{O}_{y}\right), F\right)=\operatorname{Ext}^{\bullet}\left(\mathscr{E}_{y}, F\right) \\
& =H^{p}\left(X, \mathscr{E}_{y}^{*} \otimes F\right)=H^{p}\left(X, \mathscr{E}_{y} \otimes F(-1)\right)=0
\end{aligned}
$$

по лемме 3.3 и следствию 3.6. Следовательно, $\Phi_{1}^{!}(F)=0$, так как $\left\{\mathscr{O}_{y}\right\}_{y \in C^{\vee}}-$ порождающий класс в $\mathscr{D}^{b}\left(C^{\vee}\right)$. Значит,

$$
\operatorname{Ext}^{\bullet}\left(\Phi_{1}(G), F\right)=\operatorname{Ext}^{\bullet}\left(G, \Phi_{1}^{!}(F)\right)=\operatorname{Ext}^{\bullet}(G, 0)=0
$$

для всех $G \in \mathscr{D}^{b}\left(C^{\vee}\right)$, т.е. набор $\left\langle\mathscr{U}_{+}, \mathscr{O}_{X}, \Phi_{1}\left(\mathscr{D}^{b}\left(C^{\vee}\right)\right)\right\rangle$ полуортогонален в $\mathscr{D}^{b}(X)$.

Остается проверить, что категория $\mathscr{D}^{b}(X)$ порождается $\mathscr{U}_{+}, \mathscr{O}_{X}$ и $\Phi_{1}\left(\mathscr{D}^{b}\left(C^{\vee}\right)\right)$ как триангулированная категория. Действительно, пусть $F \in{ }^{\perp}\left\langle\mathscr{U}_{+}, \mathscr{O}_{X}, \Phi_{1}\left(\mathscr{D}^{b}\left(C^{\vee}\right)\right)\right\rangle$. Так как $F \in{ }^{\perp} \Phi_{1}\left(\mathscr{D}^{b}\left(C^{\vee}\right)\right)$ имеем $\Phi_{1}^{*}(F)=0$. С другой стороны, так как $F \in{ }^{\perp}\left\langle\mathscr{U}_{+}, \mathscr{O}_{X}\right\rangle$ по предложению 4.3 имеем

$$
\Phi_{2}^{*} \circ \alpha^{*}(F)=\beta_{*} \circ \Phi_{1}^{*}(F)=0 .
$$

Однако $\Phi_{2}$ - эквивалентность по следствию 4.2, следовательно, $\Phi_{2}^{*}-$ эквивалентность; значит, $\alpha^{*}(F)=0$.

Теперь заметим, что зафиксировав $X$ (а следовательно, и $C^{\vee}$ ), и варьируя подпространство $A_{8}$, мы можем получить в качестве $S$ любое гладкое гиперплоское сечение многообразия $X$. Приведенные вьше рассуждения показывают, что ограничение всякого $F \in{ }^{\perp}\left\langle\mathscr{U}_{+}, \mathscr{O}_{X}, \Phi_{1}\left(\mathscr{D}^{b}\left(C^{\vee}\right)\right)\right\rangle \subset \mathscr{D}^{b}(X)$ на любое гладкое гиперплоское сечение изоморфно нулю.

Доказательство завершается следующей леммой.

Лемма 4.5. Если $X$ - гладкое алгебраическое многообразие, а $F$ - комплекс когерентных пучков на $X$, ограничение которого на любое гладкое гиперплоское сечение многообразия $X$ ациклично, то $F$ и сам ацикличен.

ДокАЗАТЕЛЬСтво. Предположим, что $F$ не ацикличен, и пусть $k$ - максимальное целое число такое, что $\mathscr{H}^{k}(F) \neq 0$. Выберем точку $x \in X$ в носителе пучка $\mathscr{H}^{k}(F)$. Выберем гладкое гиперплоское сечение $j: S \subset X$, проходящее через $x$. Поскольку функтор ограничения $j^{*}$ точен справа, ясно, что $\mathscr{H}^{k}\left(L j^{*} F\right) \neq 0,-$ противоречие.

5. Применение: поверхность Фано многообразия $V_{12}$. Обозначим через $F_{X}$ поверхность Фано многообразия $X$, параметризующую коники (рациональные кривые степени 2) на $X$.

Лемма 5.1. Если $R \subset X-$ коника, то

$$
\mathscr{U}_{+\mid R} \cong \mathscr{O}_{R} \oplus \mathscr{O}_{R}(-1)^{\oplus 4}
$$


ДокАЗАТЕльство. Так как $\mathscr{U}_{+}-$подрасслоение тривиального расслоения $V \otimes \mathscr{O}_{X}$ и так как $r\left(\mathscr{U}_{+\mid R}\right)=5, \operatorname{deg}\left(\mathscr{U}_{+\mid R}\right)=-4$, имеем

$$
\mathscr{U}_{+\mid R} \cong \bigoplus_{j=1}^{5} \mathscr{O}_{R}\left(-u_{j}\right), \quad \text { где } u_{j} \geqslant 0, \quad \sum u_{j}=4 .
$$

Сразу видно, что одно из $u_{j}$ равно нулю, и надо лишь показать, что такое $u_{j}$ ровно одно, т.е., что $\operatorname{dim} H^{0}\left(R, \mathscr{U}_{+\mid R}\right)=1$.

Предположим, что $\operatorname{dim} H^{0}\left(R, \mathscr{U}_{+\mid R}\right) \geqslant 2$. Выберем двумерное подпространство

$$
U \subset H^{0}\left(R, \mathscr{U}_{+\mid R}\right) \subset H^{0}\left(R, V \otimes \mathscr{O}_{R}\right)=V
$$

и рассмотрим $V^{\prime}=U^{\perp} / U$. Тогда $\operatorname{LGr}_{+}\left(V^{\prime}\right) \subset \operatorname{LGr}_{+}(V)$, и ясно, что $R \subset X^{\prime}:=$ $\mathrm{LGr}_{+}\left(V^{\prime}\right) \cap X$. Поскольку $X$ является линейным сечением грассманиана $\mathrm{LGr}_{+}(V)$, то $X^{\prime}$ является линейньм сечением $\mathrm{LGr}_{+}\left(V^{\prime}\right)$. Однако $V^{\prime}=\mathbb{C}^{6} ;$ следовательно, $\mathrm{LGr}_{+}\left(V^{\prime}\right)$ $\cong \mathbb{P}^{3}$ и, так как всякое линейное сечение $\mathbb{P}^{3}$, содержащее конику, содержит также и плоскость $\mathbb{P}^{2}$, то $X^{\prime}$ содержит $\mathbb{P}^{2}$. Следовательно, $X$ содержит $\mathbb{P}^{2}$, что невозможно по теореме Лефшеца о гиперплоском сечении, примененной к $\mathrm{LGr}_{+}(V)$ семь раз.

ЛЕмма 5.2. Выполнено равенство

$$
\bigcap_{y \in C^{\vee}} \mathscr{U}_{-y}=0
$$

ДокАЗАТЕЛЬСТво. Предположим, что $0 \neq v \subset \bigcap_{y \in C^{\vee}} \mathscr{U}_{-y}$, и рассмотрим $V^{\prime \prime}=$ $v^{\perp} / \mathbb{C} v$. Тогда $C^{\vee} \subset \mathrm{LGr}_{-}\left(V^{\prime \prime}\right) \subset \mathrm{LGr}_{-}(V)$. Более того, так как $C^{\vee}$ является линейным сечением грассманиана $\mathrm{LGr}_{-}(V)$, то $C^{\vee}$ является линейньм сечением $\mathrm{LGr}_{-}\left(V^{\prime \prime}\right)$. Далее, так как $V^{\prime \prime}=\mathbb{C}^{8}$, то $\mathrm{LGr}_{-}\left(V^{\prime \prime}\right)-$ квадрика. Кривая, являющаяся линейньм сечением квадрики, - это коника или прямая, но $C^{\vee}$ не является ни прямой, ни коникой.

ТеОРема 5.3 [10]. Выполнено равенство

$$
F_{X} \cong S^{2} C^{\vee}
$$

ДокАЗАТЕЛЬСтво. Пусть $R \subset X$ - коника. Рассмотрим разложение ее структурного пучка относительно полуортогонального разложения

$$
\mathscr{D}^{b}(X)=\left\langle\mathscr{O}_{X}, \mathscr{U}_{+}^{*}, \Phi_{1}\left(\mathscr{D}^{b}\left(C^{\vee}\right)\right)\right\rangle,
$$

полученного из разложения (8) перестройкой $\mathscr{U}_{+}$через $\mathscr{O}_{X}$. Для этого вычислим

$$
\begin{aligned}
\operatorname{Ext}^{p}\left(\mathscr{O}_{R}, \mathscr{O}_{X}\right) & =H^{3-p}\left(R, \omega_{R}\right)^{*}= \begin{cases}\mathbb{C}, & \text { если } p=2, \\
0 & \text { иначе, }\end{cases} \\
\operatorname{Ext}^{p}\left(\mathscr{O}_{R}, \mathscr{U}_{+}\right) & =\operatorname{Ext}^{3-p}\left(\mathscr{U}_{+}, \omega_{R}\right)^{*}=H^{3-p}\left(R, \mathscr{U}_{+}^{*} \otimes \omega_{R}\right)^{*} \\
& =H^{p-2}\left(R, \mathscr{U}_{+\mid R}\right)= \begin{cases}\mathbb{C}, & \text { если } p=2, \\
0 & \text { иначе, }\end{cases}
\end{aligned}
$$

по лемме 5.1. Следовательно, разложение сводится к точной последовательности

$$
0 \rightarrow \mathscr{O}_{X} \rightarrow \mathscr{U}_{+}^{*} \rightarrow \Phi_{1}\left(\Phi_{1}^{!}\left(\mathscr{O}_{R}\right)\right) \rightarrow \mathscr{O}_{R} \rightarrow 0,
$$

где

$$
\Phi_{1}^{!}: \mathscr{D}^{b}(X) \rightarrow \mathscr{D}^{b}\left(C^{\vee}\right), \quad \Phi_{1}^{!}(\cdot)=R q_{1_{*}}\left(L p_{1}^{*}(-) \otimes \mathscr{E}_{1}^{*}\left(H_{C^{\vee}}\right)\right)[1]
$$

- правьй сопряженный к $\Phi_{1}$ функтор. 
ЛЕМмА 5.4. Объект $\Phi_{1}^{!}\left(\mathscr{O}_{R}\right)$ - чистый пучок.

ДоКАЗАТЕЛЬСТВо. Чтобы описать объект

$$
\Phi_{1}^{!}\left(\mathscr{O}_{R}\right)=R q_{1_{*}}\left(L p_{1}^{*}\left(\mathscr{O}_{R}\right) \otimes \mathscr{E}_{1}^{*}\left(H_{C^{\vee}}\right)\right)[1] \in \mathscr{D}^{b}\left(C^{\vee}\right)
$$

рассмотрим

$$
H^{\bullet}\left(X, \mathscr{E}_{1}^{*}{ }_{y} \otimes \mathscr{O}_{R}\right)=H^{\bullet}\left(R, \mathscr{E}_{1}^{*}{ }_{y \mid R}\right)
$$

для всех $y \in C^{\vee}$. Пучок $\mathscr{E}_{1}^{*}$ в силу (3) является подпучком тривиального расслоения $\mathscr{U}_{-y} \otimes \mathscr{O}_{X}$, следовательно,

$$
H^{0}\left(R, \mathscr{E}_{1}^{*}{ }_{\mid R}\right) \subset \mathscr{U}_{-y} \subset V
$$

С другой стороны, по (4) пучок $\mathscr{E}_{1 y}^{*}=\mathscr{E}_{1 y}\left(-H_{X}\right)$ является подпучком в $\mathscr{U}_{+}$, следовательно,

$$
H^{0}\left(R, \mathscr{E}_{1}^{*}{ }_{y \mid R}\right) \subset H^{0}\left(R, \mathscr{U}_{+\mid R}\right)=\mathbb{C} \subset V .
$$

Значит, если $H^{0}\left(R, \mathscr{E}_{1}^{*}{ }_{\mid R}\right) \neq 0$ при всех $y \in C^{\vee}$, то $\bigcap_{y \in C} \vee \mathscr{U}_{-} \neq 0$, что противоречит лемме 5.2. Значит, для общей точки $y \in C^{\vee}$ имеем $H^{0}\left(R, \mathscr{E}_{1}^{*} y_{\mid R}\right)=0$, следовательно,

$$
R^{0} q_{1_{*}}\left(L p_{1}^{*}\left(\mathscr{O}_{R}\right) \otimes \mathscr{E}_{1}^{*}\left(H_{C^{\vee}}\right)\right)=0
$$

С другой стороны, так как $R$ - одномерное многообразие, имеем

$$
R^{k} q_{1 *}\left(L p_{1}^{*}\left(\mathscr{O}_{R}\right) \otimes \mathscr{E}_{1}^{*}\left(H_{C^{\vee}}\right)\right)=0 \quad \text { при } \quad k \neq 0,1
$$

Следовательно, $\Phi_{1}^{!}\left(\mathscr{O}_{R}\right)$ - чистьй пучок.

СлЕДСТВИЕ 5.5. Объект $\Phi_{1}^{!}\left(\mathscr{O}_{R}\right)$ - артинов пучок длины 2 на $C^{\vee}$.

ДокАЗАТЕЛЬСТво состоит в прямом вычислении характера Черна пучка $\Phi_{1}^{!}\left(\mathscr{O}_{R}\right)$ с помощью теоремы Римана-Роха-Гротендика.

Таким образом, $\Phi_{1}^{!}\left(\mathscr{O}_{R}\right)$ - либо структурньй пучок подсхемы длины 2 в $C^{\vee}$, либо $\Phi_{1}^{!}\left(\mathscr{O}_{R}\right)=\mathscr{O}_{y} \oplus \mathscr{O}_{y}$ для некоторой точки $y \in C^{\vee}$. Покажем, что второй случай невозможен. Для этого нам понадобится

ЛЕмМА 5.6. Выполнено равенство

$$
\Phi_{1}^{*}\left(\mathscr{U}_{+}^{*}\right)=\mathscr{O}_{C^{\vee}}
$$


ДокАЗАТЕЛЬСТво. Ясно, что

$$
\Phi_{1}^{*}\left(\mathscr{U}_{+}^{*}\right)=R q_{1_{*}}\left(L p_{1}^{*}\left(\mathscr{U}_{+}^{*}\right) \otimes \mathscr{E}_{1}^{*}\left(-H_{X}\right)\right)[3]=R q_{1_{*}}\left(\mathscr{E}_{1} \otimes \mathscr{U}_{+}^{*}\left(-2 H_{X}-H_{C^{\vee}}\right)\right)[3] .
$$

С другой стороны, домножая последовательность $(4)$ на $\mathscr{U}_{+}^{*}\left(-H_{X}\right)$, получаем точную последовательность

$$
\begin{aligned}
0 & \rightarrow \mathscr{E}_{1} \otimes \mathscr{U}_{+}^{*}\left(-2 H_{X}-H_{C}^{\vee}\right) \rightarrow \mathscr{U}_{+} \otimes \mathscr{U}_{+}^{*}\left(-H_{X}\right) \\
& \rightarrow \mathscr{U}_{-}^{*} \otimes \mathscr{U}_{+}^{*}\left(-H_{X}\right) \rightarrow \mathscr{E}_{1} \otimes \mathscr{U}_{+}^{*}\left(-H_{X}\right) \rightarrow 0 .
\end{aligned}
$$

Из леммы 3.3 следует, что $R^{\bullet} q_{1_{*}}\left(\mathscr{E}_{1} \otimes \mathscr{U}_{+}^{*}\left(-H_{X}\right)\right)=0$, а следствие 3.2 означает, что

$$
\begin{aligned}
& R^{\bullet} q_{1_{*}}\left(\mathscr{U}_{-}^{*} \otimes \mathscr{U}_{+}^{*}\left(-H_{X}\right)\right)=0, \\
& R^{k} q_{1_{*}}\left(\mathscr{U}_{+} \otimes \mathscr{U}_{+}^{*}\left(-H_{X}\right)\right)= \begin{cases}\mathscr{O}_{C} & \text { при } k=3, \\
0 & \text { при } k \neq 3,\end{cases}
\end{aligned}
$$

и лемма вытекает из спектральной последовательности Лере.

ЛЕмма 5.7. Выполнено условие

$$
\Phi_{1}^{!}\left(\mathscr{O}_{R}\right) \neq \mathscr{O}_{y} \oplus \mathscr{O}_{y}
$$

ДокАЗАТЕЛЬСтво. Если бы имело место равенство, то разложение (9) имело бы вид

$$
0 \rightarrow \mathscr{O}_{X} \rightarrow \mathscr{U}_{+}^{*} \rightarrow \mathscr{E}_{1 y} \oplus \mathscr{E}_{1 y} \rightarrow \mathscr{O}_{R} \rightarrow 0
$$

С другой стороны, из леммы 5.6 следует, что

$$
\begin{aligned}
\operatorname{Hom}\left(\mathscr{U}_{+}^{*}, \mathscr{C}_{1 y}\right) & =\operatorname{Hom}\left(\mathscr{U}_{+}^{*}, \Phi_{1}\left(\mathscr{O}_{y}\right)\right) \\
& =\operatorname{Hom}\left(\Phi_{1}^{*}\left(\mathscr{U}_{+}^{*}\right), \mathscr{O}_{y}\right)=\operatorname{Hom}\left(\mathscr{O}_{C \vee}, \mathscr{O}_{y}\right)=\mathbb{C},
\end{aligned}
$$

следовательно, ранг всякого отображения $\mathscr{U}_{+}^{*} \rightarrow \mathscr{E}_{1 y} \oplus \mathscr{E}_{1 y}$ не превосходит 2 , и такая точная последовательность невозможна.

СлЕДСтвиЕ 5.8. Оббект $\Phi_{1}^{!}\left(\mathscr{O}_{R}\right)$ - структурный пучок подсхемы длины 2 в $C^{\vee}$. Функтор $\Phi_{1}^{!}$индуцирует отображение $F_{X} \rightarrow S^{2} C^{\vee}$.

Обратно, если $Z$ - подсхема длины 2 в $C^{\vee}$, то

$$
\mathbb{C}=\operatorname{Hom}\left(\mathscr{O}_{C}, \mathscr{O}_{Z}\right)=\operatorname{Hom}\left(\Phi_{1}^{*}\left(\mathscr{U}_{+}^{*}\right), \mathscr{O}_{Z}\right)=\operatorname{Hom}\left(\mathscr{U}_{+}^{*}, \Phi_{1}\left(\mathscr{O}_{Z}\right)\right) .
$$

Следовательно, существует нетривиальньй гомоморфизм $f: \mathscr{U}_{+}^{*} \rightarrow \Phi_{1}\left(\mathscr{O}_{Z}\right)$. Его ядро является рефлексивньм пучком ранга 1 с $c_{1}=0$, следовательно, изоморфно пучку $\mathscr{O}_{X}$, и несложно показать, что коядро этого гомоморфизма есть структурньй пучок коники. Следовательно, отображение $\Phi_{1}^{!}: F_{X} \rightarrow S^{2} C^{\vee}$ является изоморфизмом.

Автор выражает признательность Атанасу Илиеву и Дмитрию Маркушевичу за важные сведения о многообразиях $V_{12}$, а также Дмитрию Орлову и Алексею Бондалу за полезные обсуждения. 


\section{СПИСОК ЦИТИРОВАННОЙ ЛИТЕРАТУРЫ}

[1] Kuznetsov A. G. Fano threefolds V22 // Preprint MPI 1997-24. Bonn: MPI, 1997.

[2] Кузнецов А.Г. Производная категория трехмерной кубики и многообразия $V_{14} / / \mathrm{Tp}$. МИАН. 2004. Т. 246. С. 183-207.

[3] Mukai S. Fano 3-folds // Complex Projective Geometry (Trieste, 1989/Bergen, 1989). London Math. Soc. Lecture Notes Ser. V. 179. Cambridge: Cambridge Univ. Press, 1992. P. 255-263.

[4] Iliev A., Markushevich D. Elliptic curves and rank 2 vector bundles on the prime Fano threefolds of genus 7 // Adv. Geom. 2004. V. 4. № 3. P. 287-318.

[5] Bridgeland T. Equivalences of triangulated categories and Fourier-Mukai transforms // Bull. London Math. Soc. 1999. V. 31. № 1. P. 25-34.

[6] Исковских В. А. Трехмерные многообразия Фано. I // Изв. АН СССР. Сер. матем. 1977. T. 41. С. 516-562; II // Изв. АН CСCР. Сер. матем. 1978. Т. 42. С. 506-549.

[7] Iskovskikh V. A., Prokhorov Yu. G. Algebraic Geometry V: Fano Varieties. Encyclopaedia Math. Sci. V. 47. Berlin: Springer-Verlag, 1998.

[8] Demazure M. A very simple proof of Bott's theorem // Invent. Math. 1976. V. 33. №3. P. 271-272.

[9] Bondal A., Orlov D. Semiorthogonal decompositions for algebraic varieties // Preprint MPI 1995-15; E-print math. AG/9506012. Bonn: MPI, 1995.

[10] Iliev A., Markushevich D. Parametrization of $\operatorname{Sing}(\Theta)$ for a Fano 3-fold of genus 7 by moduli of vector bundles // E-print math. AG/0403122, 2004.

Математический институт им. В. А. Стеклова, РАН

Поступило

E-mail: akuznet@mi.ras.ru, sasha@kuznetsov.mccme.ru

22.11 .2004 